\title{
Inertial fusion energy target output and chamber response: Calculations and experiments ${ }^{\text {a) }}$
}

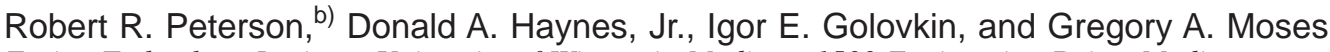 \\ Fusion Technology Institute, University of Wisconsin-Madison, 1500 Engineering Drive, Madison, \\ Wisconsin 53706
}

(Received 29 October 2001; accepted 31 January 2002)

\begin{abstract}
The emission of photons and energetic ions by the burn and subsequent explosion of inertial fusion energy (IFE) targets poses a threat to the survival of the target chambers in future IFE power plants. Immediately after the deposition of target output, the chamber can experience sufficient heating to cause vaporization, melting, and shock loading on chamber walls. Until high-yield targets can be ignited in laboratory experiments, predictions of the nature of the target output and the response of the target chamber must be made with radiation-hydrodynamics computer codes that need to be validated with relevant smaller scale experiments. Physical models of equation of state, opacity, and radiation transport are in special need of validation. Issues of target output and chamber response requiring experiments and computer modeling are discussed and initial results from experiments are presented. Calculations of $\mathrm{x}$ ray and debris output from direct-drive IFE targets are shown and sensitivity of the output spectra and chamber response to details of the physics models are discussed.

(C) 2002 American Institute of Physics. [DOI: 10.1063/1.1464543]
\end{abstract}

\section{INTRODUCTION}

In inertial fusion energy (IFE), a fusion fuel capsule explosively burns, emitting $\mathrm{x}$ rays, $\gamma$ 's, neutrons, ions, and electrons. For economically viable IFE, the explosion must occur in some type of target chamber designed to survive $10^{8}$ such explosions ${ }^{1}$ in a year of operation while efficiently recovering the energy released and the target remnants. The chamber must be designed to recover from the explosion to allow target injection and IFE driver beam (laser or ion) transport rapidly enough to allow a repetition rate of several $\mathrm{Hz}$. The need for chamber survival of very many explosions and high repetition rate distinguishes IFE from the inertial confinement fusion (ICF) technologies being developed in programs such as the National Ignition Facility (NIF). In NIF, several very low-yield shots a day are expected initially, and once moderate yield $(\sim 10 \mathrm{MJ})$ is attained, substantially longer times between shots may occur. Before a demonstration IFE plant can be considered, integrated research experiments (IRE) would be built and operated in part to study target chamber response issues. Target output experiments will be possible on NIF once target ignition is achieved.

Prior to design of IREs, computer calculations and experiments are in progress to assess what target and chamber concepts are promising candidates for IFE. The chamber concepts can be segregated into three types: dry wall, wetted wall, and thick liquid wall. The targets are either direct drive or indirect drive. There is also the possibility of fast-ignition targets of either type. In this paper, we discuss calculations for laser direct-drive target output in a dry-wall chamber. In these calculations, we have used a target concept developed at the Naval Research Laboratory. ${ }^{2,3}$ This target concept consists of a shell of cryogenically cooled deuterium-tritium

\footnotetext{
${ }^{a)}$ Paper CI1 6, Bull. Am. Phys. Soc. 46, 51 (2001).

${ }^{b}$ Invited speaker. Electronic mail: rrpeter@engr.wisc.edu
}

(DT) fuel, surrounded by an ablator made of plastic foam wetted with liquid DT, which is then frozen. The ablator is covered with a $1 \mu \mathrm{m}$ thick plastic shell that is itself covered with several hundred $\AA$ of gold or palladium. In this concept, the gold or palladium is heated by the $0.25 \mu \mathrm{m} \mathrm{KrF}$ laser light to the point where energy from the gold or palladium is radiated into the plastic shell and ablator. The radiation heating limits the growth of Rayleigh-Taylor fluid instabilities and smoothes laser imprinting in the ablator. ${ }^{4,5}$

In this work, the explosion of this target is modeled with the BUCKY ${ }^{6}$ computer code, where the output of the target is predicted. Other codes have been used at the Naval Research Laboratory and Lawrence Livermore National Laboratory to design these targets. It is useful to duplicate those calculations by starting BUCKY simulations at the beginning of laser turn-on and modeling the implosion and burn of the target, thereby producing the detailed plasma conditions of the target at the time of ignition. The explosion is assumed to occur in a graphite dry-wall target chamber filled with xenon gas, as designed in the SOMBRERO study. ${ }^{1}$ The target chamber gas can be designed to stop enough of the target $\mathrm{x}$ rays and ions and reradiate the energy over a long enough time that the graphite does not sublime. Computer calculations show how sensitive the design is to details in the target output. Finally, experiments are in progress on the Z-machine at Sandia National Laboratories, where $\mathrm{x}$ rays similar in fluence and spectrum to IFE target $\mathrm{x}$ rays are being used to vaporize possible target chamber materials. ${ }^{7}$ These experiments are being used to validate the BUCKY code's ability to model these phenomena.

\section{CALCULATIONAL METHODS}

The BUCKY computer code has been used to calculate IFE target output, IFE chamber dynamics, and the vaporiza- 
tion of sample materials in Z-machine experiments. BUCKY is a one-dimensional Lagrangian radiation-hydrodynamics computer code. The hydrodynamic fluid is assumed to be quasineutral and to obey classical hydrodynamics. Fluxlimited multigroup radiation diffusion is used to model radiation transport. Multigroup opacities are found in electron temperature versus ion density tables produced by the IONMIX and EOSOPA ${ }^{8}$ computer codes. Equations of state are also found in tables produced by these two codes or SESAME tables from Los Alamos National Laboratory. Electron and ion temperatures are calculated separately and thermal conduction is calculated for both within a flux-limited diffusion model. Both species are assumed to be in Maxwellian velocity distributions.

Laser deposition is calculated in full geometric generality, with programmed zooming ${ }^{9}$ possible. The laser energy is propagated along rays that deposit their energy and are reflected and refracted as dictated by the local electron density. An inverse Bremsstrahlung model is used for the energy deposition model. The model turns or fully absorbs all laser rays before they pass through the critical surface.

Thermonuclear burn of the DD, DT, and $\mathrm{DHe}^{3}$ is calculated based on fuel densities and tabulated ion temperaturedependent rates. Ionic fusion products are tracked with their times of flight included and their energy deposited along the tracks to both electrons and ions. Neutron energy is redeposited with an escape probability method, which does not take spectral changes into account. Depletion of the fuel is taken into account.

Vaporization and melting of target chamber materials can be treated outside of the Lagrangian hydrodynamics mesh. Energy is exchanged between the hydrodynamic mesh and a surrounding mesh that includes no hydrodynamic motion but does include thermal conduction and the deposition of ions, photons, and electrons. The outer mesh also models phase transitions (melting, vaporization, or sublimation, and condensation). When a zone in the outer mesh is determined to vaporize or sublime by one of a few models based on temperature and the density in the adjacent Lagrangian mesh, that zone becomes part of the Lagrangian mesh and can undergo hydrodynamic motion. When these models dictate that the outermost zone in the Lagrangian mesh should condense, the inverse occurs. Latent heats are included in all phase transitions. Temperature-dependent thermal properties are used in the outer mesh.

Equations-of-state and multigroup opacity tables are prepared by the IONMIX and EOSOPA computer codes. The IONMIX code uses hydrogenic atomic models supplemented by detailed multielectron ground-state ionization potentials for atomic energy level structures and cross sections for collisional and radiative properties. It solves a collisional radiative equilibrium (CRE) model to determine ionization state and atomic level populations, interpolating between coronal and collisional equilibria. EOSOPA generates detailed multielectron atomic data for all ionization stages by solving the Hartree-Fock equations with relativistic corrections. The Saha equilibrium model is used to generate the ionization state and unresolved transition array (UTA) method for level structure. Both codes provide the average charge states used

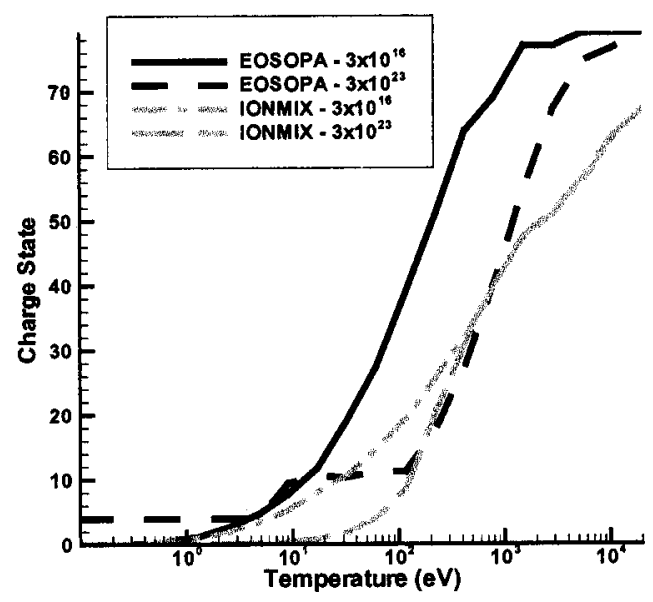

FIG. 1. Average charge state for gold calculated with EOSOPA and IONMIX.

in BUCKY. The average charge states predicted for gold by the two codes are shown in Fig. 1. At high density one sees that IONMIX is less appropriate because there is no pressure ionization and the charge state is very low even up to $30 \mathrm{eV}$. On the other hand, EOSOPA uses the Saha model far outside of its region of validity, which is at low density and high temperature. It predicts charge states that are very high in the coronal regime because it assumes that three-body recombination, which is rare at low density, is in balance with collisional ionization. Therefore, one must be careful to use IONMIX and EOSOPA only where they are valid.

\section{IFE LASER TARGET OUTPUT}

We have performed BUCKY calculations for the $\mathrm{KrF}$ laser-driven implosion, burn, and explosion of an NRL radiation-smoothed target. We have performed calculations with IONMIX and EOSOPA opacities used for the thin gold and palladium layers. The laser has been zoomed twice, at 29.8 and $32.1 \mathrm{~ns}$ in a $34 \mathrm{~ns}$ pulse. Each time zooming is done the laser beams are narrowed: from $0.244 \mathrm{~cm}$ in radius, to 0.179 $\mathrm{cm}$, and finally to $0.131 \mathrm{~cm}$. When the laser is zoomed the intensity is increased as $\left(R_{0} / R\right)^{0.9}$, so some, but not all, of the laser energy is compressed into the narrower beams. The total laser power versus time is shown in Fig. 2. The total laser power is shown for both zoomed and unzoomed cases. Zooming reduces the deposited laser energy to $2.33 \mathrm{MJ}$. The resulting target yield is about $350 \mathrm{MJ}$. The gold target outer radius is $2.43973 \mathrm{~mm}$, the ablator outer radius is 2.43870 $\mathrm{mm}$, the fuel outer radius is $2.1125 \mathrm{~mm}$, and the fuel inner radius is $1.875 \mathrm{~mm}$. The inner cavity contains DT gas at 0.3 $\mathrm{mg} / \mathrm{cm}^{3}$. The fuel mass is $2.976 \mathrm{mg}$. The palladium-coated target is the same, except the palladium is $1200 \AA$ thick.

The details of the implosion for a palladium target are shown in Fig. 3, where the positions of Lagrangian zone boundaries are plotted against time. One can clearly see the ablator expanding due to the radiative preheating prior to the main shock. This will provide ablative stabilization of Rayleigh-Taylor instabilities. This calculation used IONMIX opacities for palladium and provided $356 \mathrm{MJ}$ of yield. The same calculation using an EOSOPA opacity for the palladium 


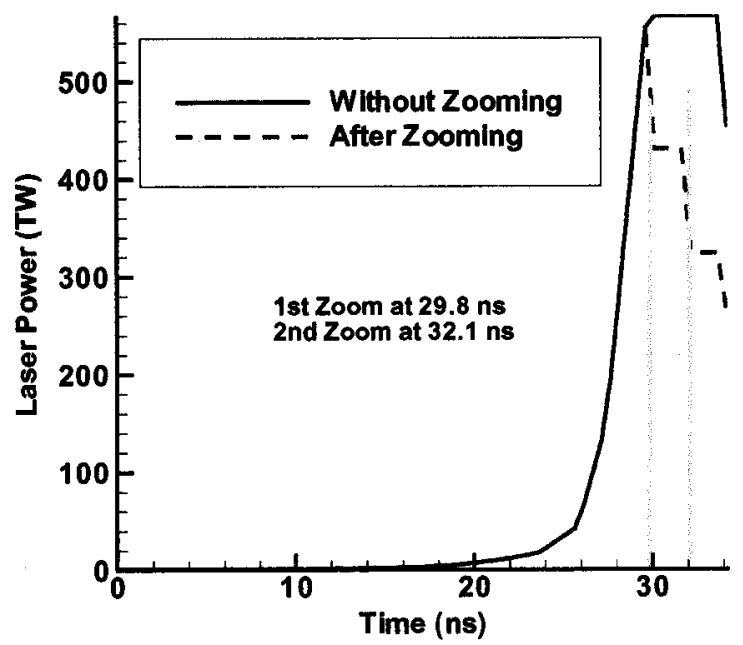

FIG. 2. Total laser power versus time with and without zooming.

gives very similar yield, though the ablator expansion due to radiative preheat is earlier. The target output for both targets, using EOSOPA and IONMIX opacity tables for the gold and palladium, are shown in Table I. The yield and energy partition are remarkably similar for all cases except the EOSOPA case for gold. This particular case radiated more strongly from the high- $\mathrm{Z}$ shell both before and after ignition, which affected the implosion, the yield, and the energy partition. This is in agreement with other work, ${ }^{10}$ which shows that collisional radiative equilibrium (CRE) and local thermodynamic equilibrium (LTE) treatments of the gold opacity in slab experiments lead to different hydrodynamic behavior.

The time-integrated output $\mathrm{x}$-ray spectra for similar target calculations, using IONMIX and EOSOPA opacities for gold and palladium, are shown in Fig. 4. Once again, the two palladium cases and the IONMIX calculation for gold give very similar results, while the gold spectrum for EOSOPA is much higher for low photon energies. The differences can be explained by comparing curves in Fig. 1. The charge state of the gold below $100 \mathrm{eV}$ and at high density, as it is in the initial phases of laser irradiation, is quite different. The

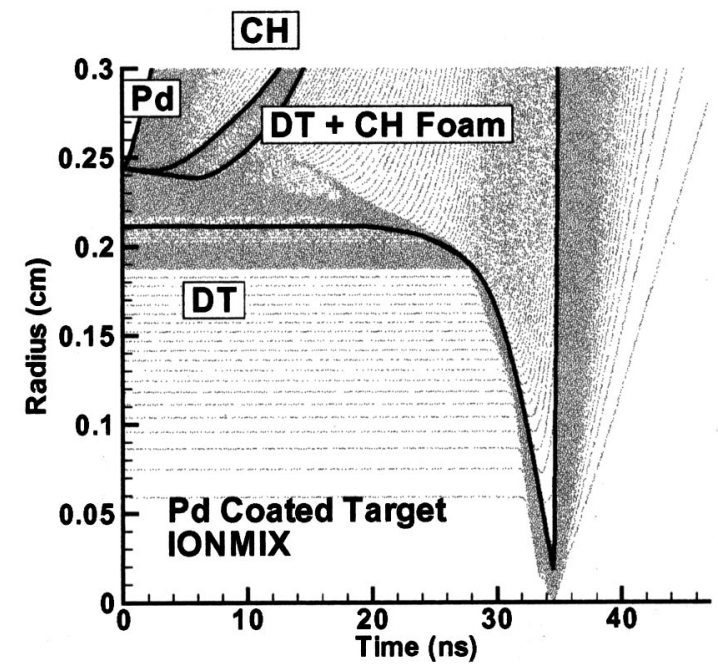

FIG. 3. Radius versus time plot for NRL 350 MJ target using IONMIX opacities.
TABLE I. Target yield and energy partition for gold and palladium directdrive laser targets.

\begin{tabular}{lllll}
\hline \hline & Au EOSOPA & Au IONMIX & Pd EOSOPA & Pd IONMIX \\
\hline Yield (MJ) & 281.1 & 353.1 & 353.7 & 355.7 \\
& $(99.0 \%)$ & $(99.2 \%)$ & $(99.2 \%)$ & $(99.2 \%)$ \\
Neutron (MJ) & 209.6 & 257.0 & 256.7 & 260.1 \\
& $(73.8 \%)$ & $(72.2 \%)$ & $(72.0 \%)$ & $(72.5 \%)$ \\
X ray (MJ) & 4.94 & 2.66 & 2.68 & 2.71 \\
& $(1.74 \%)$ & $(0.75 \%)$ & $(0.75 \%)$ & $(0.76 \%)$ \\
Target debris & 68.4 & 74.6 & 78.1 & 68.4 \\
(MJ) & $(24.8 \%)$ & $(21.0 \%)$ & $(21.9 \%)$ & $(19.1 \%)$ \\
Charged & 1.08 & 21.7 & 19.1 & 20.9 \\
fusion & $(0.38 \%)$ & $(6.1 \%)$ & $(5.4 \%)$ & $(5.8 \%)$ \\
product (MJ) & & & & \\
\hline \hline
\end{tabular}

EOSOPA has a much higher charge state and therefore, a higher electron density. The differences in the electron density change how the laser behaves and the timing of the implosion. So, the laser would have to be separately tuned for each case, which was not done. Therefore, the target yield is not the same. Shortly after ignition time, when most of the $\mathrm{x}$ rays are emitted, the outer gold shell is lower in density but very hot. Here, the EOSOPA charge state is too high, as was discussed earlier. This changes the opacity and the radiation released. It also affects the spectrum of the debris ions. What is needed is a charge state calculation that is valid everywhere in density-temperature space.

The debris ion spectrum is shown for the palladium IONMIX calculation in Fig. 5. This spectrum is obtained by recording the velocity of each Lagrangian cell $12 \mathrm{~ns}$ after ignition time and assuming that every particle in the cell has that cell's velocity. Lagrangian hydrodynamics codes like BUCKY can have difficulty resolving the pressure profiles leading to the blow-off of the outermost zone. Theoretically, a fluid should not blow off material greater than $2 /(\gamma-1)$ times sound speed where $\gamma$ is the ratio of specific heats, ${ }^{11}$ so we have demanded that constraint be met. Another worry is that the very thin gold or palladium layer is not quasineutral or does not have sufficient collisions to be considered a fluid. These are both related to collisional mean free paths for elec-

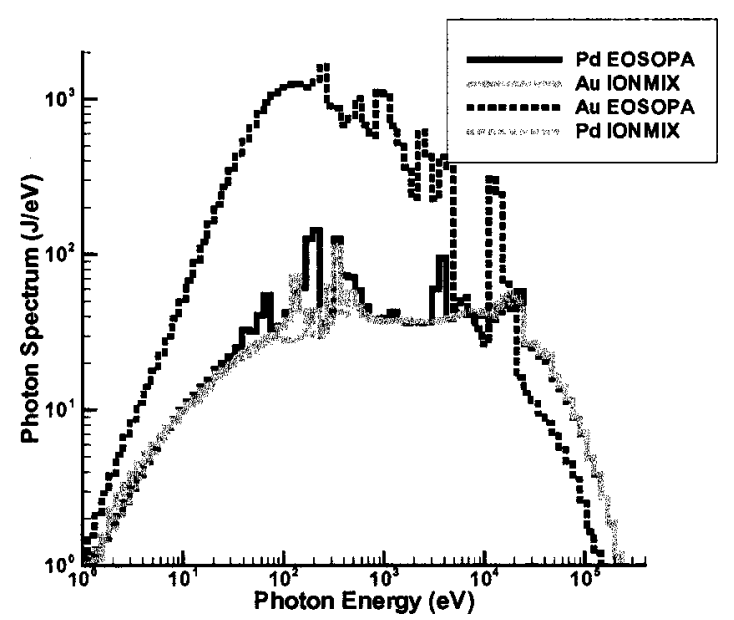

FIG. 4. Time-integrated $\mathrm{x}$-ray output from $350 \mathrm{MJ}$ NRL target as predicted by BUCKY using IONMIX and EOSOPA opacities for gold and palladium. 


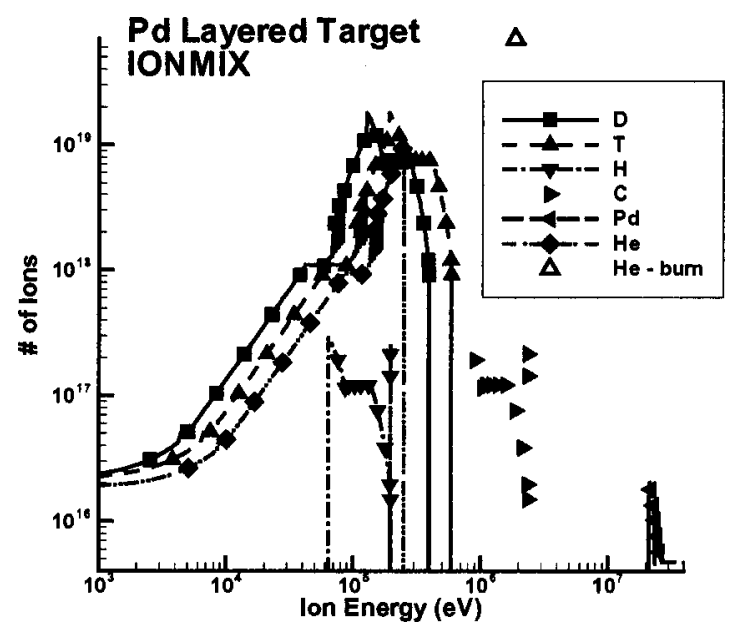

FIG. 5. Debris ion spectra from $350 \mathrm{MJ}$ NRL target as predicted by BUCKY using IONMIX opacities.

trons and ions in this layer. Calculations of these mean free paths show that they can be long compared to the size of the layer. $^{12}$

We believe that the issues related to target output will require validation experiments to be resolved. NIF ignition experiments will certainly provide some very valuable data in this regard. There are probably other experiments that can be done or have been done to mimic the plasma conditions in an exploding capsule. This was recently attempted on the Z-machine but could not reach sufficient energy densities.

\section{GAS-PROTECTED CHAMBER RESPONSE}

The target output presented in the preceding section has been used to study the response of a gas-filled, graphite-lined target chamber. The dry-wall concept uses high-temperature material such as graphite, tungsten, and silicon carbide, perhaps in conjunction with a target chamber fill gas to keep the first wall from eroding over time because of phase changes. Because the number of shots is very large, well less than one layer of atoms on the surface can be lost per shot. Using a xenon fill gas and a graphite wall $6.5 \mathrm{~m}$ in radius, we have tried to determine the minimum fill-gas density required to avoid sublimation for several target concepts.

We have used the BUCKY code in these calculations. BUCKY calculates the time-dependent debris ion deposition in the gas and wall. Target $\mathrm{x}$ rays are also calculated in the gas and wall using cold multigroup stopping powers in conjunction with hot opacities from IONMIX and EOSOPA. The energy that is stopped in the gas heats the xenon to the point that it radiates much of its energy to the wall, but over a time that is long compared to the original deposition time and, hopefully, the temperature diffusion time in the wall. For the gas to prevent wall damage, it must be at least as thick as the range of the ions and $\mathrm{x}$ rays. It is important to consider time-offlight spreading of the ions and non-LTE radiation transport in modeling the gas and wall response. ${ }^{13}$ In the calculations presented here, flux-limited radiation diffusion is used even though it has been shown for some cases that non-LTE transport is required. ${ }^{13}$ An examination into this issue is planned for future work.

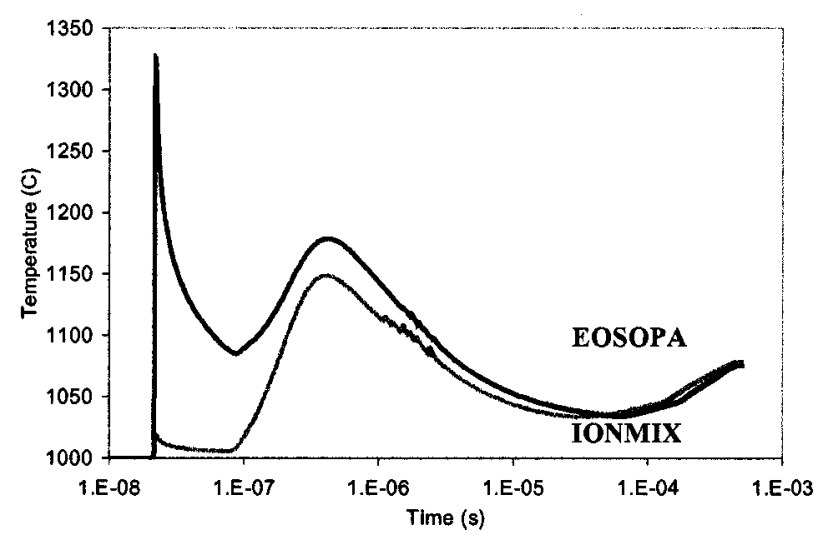

FIG. 6. Graphite wall surface temperature versus time for $25 \mathrm{mtorr}, 6.5 \mathrm{~m}$ radius target chambers as predicted by BUCKY using target output from BUCKY using IONMIX opacities for gold (392 MJ) and EOSOPA opacities for gold (263 MJ).

We specifically tested the role of the target output details of the gold coated target in Table I on the wall response by performing BUCKY calculations at 25 mtorr xenon density for the output calculated with IONMIX and EOSOPA opacities for gold. The temperature histories are shown for the two cases in Fig. 6. It is clear that the softer x-ray spectrum and larger energy fraction in the EOSOPA calculation more than compensates for the lower yield. The second rise in the wall-surface temperature is due to the target-produced ions. In both cases there was no erosion of the wall, but if the target threat is closer to what is predicted from using IONMIX ionization, there is more flexibility in the chamber design. This clearly makes the point how important it is to get the correct target output details.

\section{Z-MACHINE CHAMBER RESPONSE VALIDATION EXPERIMENTS}

BUCKY and other codes calculate the response of materials to $\mathrm{x}$ rays and debris ions based on physical models. These models may often be implicitly assuming ideal behavior that is not actually present in the real substance. For example, the vaporization model may implicitly assume that the material is homogeneous and symmetric, while woven composites are clearly not. To test the models in BUCKY and other codes we have begun experiments where $\mathrm{x}$ rays produced in $\mathrm{Z}$ pinches are directed onto samples. The $\mathrm{Z}$-machine is a large Z-pinch facility that produces $1.9 \mathrm{MJ}$ of $\mathrm{x}$ rays in less than $10 \mathrm{~ns}$, when $20 \mathrm{MA}$ of electrical current pinches cylindrical arrays of hundreds of tungsten wires. A team of scientists from Sandia National Laboratories, the University of California (San Diego and Berkeley campuses), ESL, Inc., and the University of Wisconsin has started to investigate wall response to $\mathrm{x}$ rays using $\mathrm{Z}$. Placing samples in collimated boxes to minimize debris from the pinch assembly, an x-ray loading of $48 \mathrm{~J} / \mathrm{cm}^{2}$ of IFE relevant $\mathrm{x}$ rays is achieved. Wire array pinches on the Z-machine produce a thermal spectrum of $x$ rays that peaks at $300 \mathrm{eV}$, while the direct-drive targets will emit photons from $100 \mathrm{eV}$ to $20 \mathrm{keV}$. The effects of spectrum will be studied by filtering and using other wire materials that emit lines in the few $\mathrm{keV}$ 
Z Shot $783 \quad$ Estimated Fluence $=42 \mathrm{~J} / \mathrm{cm}^{2}$

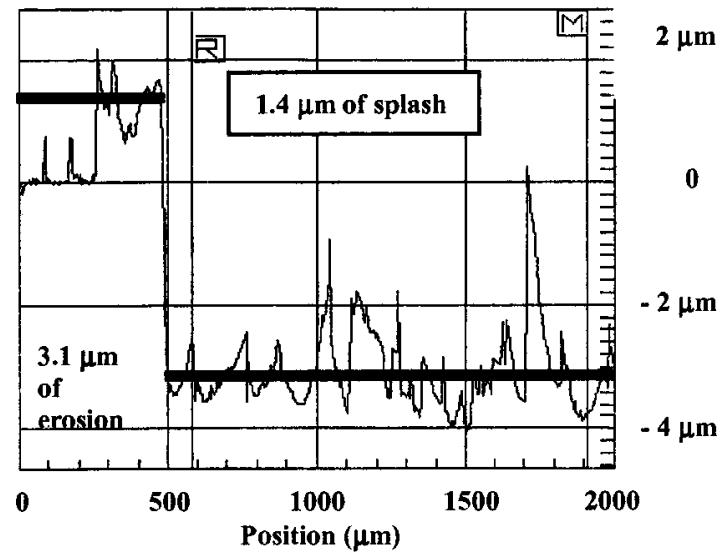

FIG. 7. Profilometry results from LiF samples irradiated on $\mathrm{Z}$ with an estimated $48 \mathrm{~J} / \mathrm{cm}^{2}$ of tungsten wire-array $x$ rays. The surface height of the irradiated sample is plotted against lateral position. Three $\mu \mathrm{m}$ of erosion is estimated.

range. These experiments only study $x$-ray damage to the samples since there are no neutrons or penetrating ions produced on the Z-machine.

Figure 7 shows the results of profilometry measurements of a lithium fluoride sample. These preliminary measurements show that about $3 \mu \mathrm{m}$ is removed and about $1.5 \mu \mathrm{m}$ is deposited in the unirradiated region. There is speculation that this build-up is due to flow of molten material. Scanning electron microscope imaging of the sample showed a roughly $4 \mu \mathrm{m}$ erosion depth. The discrepancy between these two results may be due to variations in the erosion over the surface and is still under study. Figure 8 shows the results of BUCKY calculations and a UC Berkeley estimate of vaporization, which are compared to the experimental results. A 3-D view-factor calculation ${ }^{14}$ was performed for the $\mathrm{Z}$ geometry to provide the time-dependent $\mathrm{X}$-ray spectrum on the surface of the irradiated sample. The BUCKY results predict how

\section{BUCKY Simulations of $Z$ Experiments}

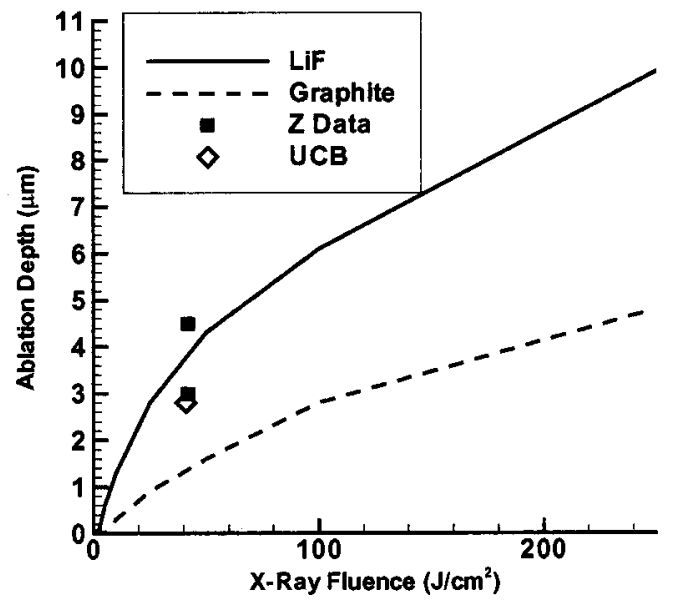

FIG. 8. BUCKY predictions of ablation depths from vaporization by $\mathrm{Z}$ produced $\mathrm{x}$ rays in graphite and lithium fluoride. Results are plotted versus $\mathrm{x}$-ray fluence. An estimate of lithium fluoride vaporization, assuming a 100 $\mathrm{eV}$ black-body spectrum of $\mathrm{x}$ rays and vaporizing only the material that exceeds the cohesive energy, was performed by the University of California Berkeley (UCB). graphite and lithium fluoride respond to varying levels of $\mathrm{x}$ rays. At $41 \mathrm{~J} / \mathrm{cm}^{2}$, BUCKY predicts $3.0 \mu \mathrm{m}$ of evaporation in lithium fluoride. Berkeley estimates $2.8 \mu \mathrm{m}$ at $41 \mathrm{~J} / \mathrm{cm}^{2}$, but with a cruder approximation for the spectrum. The BUCKY and Berkeley calculations are in agreement with the experimental values.

More experiments and calculations are required to validate our understanding of these phenomena. Experiments and BUCKY calculations are planned in the near future for different types of graphite and tungsten. The BUCKY calculations presented in Fig. 8 predict a threshold for vaporization in graphite of $5 \mathrm{~J} / \mathrm{cm}^{2}$. Experiments are planned below this fluence to study how materials irradiated below the threshold behave. The tungsten experiments will include known amounts of impurities to study that effect.

\section{CONCLUSIONS}

We have performed BUCKY code calculations of IFE target performance, target chamber response, and Z-machine $\mathrm{X}$-ray vaporization experiments. It is clear from this exercise that

(1) Ionization models need to be valid over the full range of target plasma conditions. Saha, coronal, and fully timedependent ionization models each are most appropriate for different ranges of temperature and density. Yet, the target and target chamber gases experience a wide range of plasma conditions that spans the ionization regimes.

(2) Target output is sensitive to ionization model details. The detailed radiation emission from the high- $Z$ coating on the target and the implosion are affected by how the ionization state is calculated. Full time-dependent ionization calculations have not been performed by the authors but are planned. The effect of ionization model is less pronounced in palladium coated targets than in gold coated.

(3) Target chamber response is sensitive to changes in the target output. The presence of soft $\mathrm{x}$ rays in the gold target using LTE ionization leads to a substantially different threat to the target chamber first wall and changes the chamber requirements. Therefore, the validation of the target output threat spectra is important to the drywall target chamber concept for direct-drive laser fusion.

(4) Z-machine experiments are useful in validating wallresponse models to pulsed $x$ rays. BUCKY simulations of a $\mathrm{Z}$ experiment for $\mathrm{LiF}$ predicted the amount of vaporization that agreed with the experiment. Experiments are planned that will test BUCKY predictions of the threshold for X-ray damage in graphite.

\section{ACKNOWLEDGMENTS}

The authors wish to acknowledge the help of Dr. C. L. Olson, G. A. Rochau, Dr. T. Tanaka, Dr. T. J. Renk, and M. Ulrickson of Sandia National Laboratories, and Professor P. F. Peterson at the University of California-Berkeley in the performance of Z-machine experiments. They also wish to thank Dr. J. Sethian, Dr. J. Gardner, Dr. D. Colombant, and Dr. A. Schmitt of the Naval Research Laboratory and Dr. L. 
J. Perkins of Lawrence Livermore National Laboratory for discussions related to the target output calculations. Professor G. L. Kulcinski and the rest of the University of Wisconsin Fusion Technology Institute have helped with design considerations of the gas-filled target chamber. The 3-D view factor calculations were performed by G. A. Rochau, of Sandia National Laboratories and the University of Wisconsin.

This work is supported by the Naval Research Laboratory and the US DOE.

${ }^{1}$ I. N. Sviatoslavsky, M. E. Sawan, R. R. Peterson et al., Fusion Technol. 21, 1470 (1992).

${ }^{2}$ S. E. Bodner, D. G. Colombant, J. H. Gardner et al., Phys. Plasmas 5, 1901 (1998).

${ }^{3}$ S. E. Bodner, D. G. Colombant, J. H. Gardner et al., Phys. Plasmas 7, 2298 (2000).

${ }^{4}$ S. E. Bodner, Phys. Rev. Lett. 33, 761 (1974).
${ }^{5}$ S. P. Obenschain, D. Colombant, M. Karasik et al., Phys. Plasmas 9, 2234 (2002).

${ }^{6}$ R. R. Peterson, J. J. MacFarlane, J. F. Santarius et al., Fusion Technol. 30, 783 (1996).

${ }^{7}$ R. R. Peterson, C. L. Olson, T. J. Renk, G. A. Rochau, and M. A. Sweeney, Nucl. Instrum. Methods Phys. Res. A 464, 172 (2001).

${ }^{8}$ P. Wang, Ph.D. dissertation, Department of Nuclear Engineering and Engineering Physics, University of Wisconsin, Madison, WI, 1991.

${ }^{9}$ R. H. Lehmberg and J. Goldhar, Fusion Technol. 11, 532 (1987).

${ }^{10}$ N. Ohnishi, H. Nagatomo, H. Nishimura et al., J. Quant. Spectrosc. Radiat. Transf. 71, 551 (2001).

${ }^{11}$ Ya. B. Zel'dovich and Yu. P. Raizer, Physics of Shock Waves and HighTemperature Hydrodynamic Phenomena (Academic, New York, 1966), p. 42.

${ }^{12}$ J. F. Santarius (private communication, 2001).

${ }^{13}$ J. J. MacFarlane and P. Wang, Phys. Fluids B 3, 3494 (1991).

${ }^{14}$ This calculation was performed with the VIS-RAD computer code, which is developed by Prism Computational Sciences of Madison, WI. 